\title{
Commentary
}

\section{Increased vulnerability of older adults to DM and its CVD complications: proposal for assessment, management and monitoring by diagnostic methods}

\author{
Ezekiel Uba Nwose $^{1}$, Phillip Taderera Bwititi ${ }^{2}$, Ross Stuart Richards ${ }^{1}$
}

\author{
${ }^{1}$ School of Community Health, Charles Sturt University, New South Wales, Australia \\ ${ }^{2}$ School of Biomedical Sciences, Charles Sturt University, New South Wales, Australia
}

Received: 29 January 2015

Accepted: 8 February 2015

\section{*Correspondence:}

Dr. Ezekiel Uba Nwose,

E-mail: nwoseeu@gmail.com, enwose@csu.edu.au

Copyright: () the author(s), publisher and licensee Medip Academy. This is an open-access article distributed under the terms of the Creative Commons Attribution Non-Commercial License, which permits unrestricted non-commercial use, distribution, and reproduction in any medium, provided the original work is properly cited.

\begin{abstract}
The national institute of health on aging has made research funding calls for basic, clinical and epidemiological studies into the increased vulnerability of older adults to diabetes mellitus and its cardiovascular complications. One of the indicated outcomes is the impact of age on diagnosis and management of the disease co-morbidity, including elucidation of the role of aging mechanisms that underlie the increased vulnerability of older adults. A cursory review of literature reveals that although there is information about aging and oxidative stress, little has been validated for adoption in the context of pathology-based evidence. Therefore, the objective of this hypothesis paper is to revisit the topic of 'biochemical basis of metabolism' in aging and diseases. The concepts of 'aging-induced oxidative stress' and 'antioxidant-vitamins' basis of diabetes progression' are briefly linked to propose that increase in oxidative damage is a potential additive outcome underlying increased vulnerability of older adults to diabetes mellitus and its cardiovascular complications. Further, the prospect of oxidative damage indices is re-presented as a hypothesis for validation to provide pathology evidence-based guidance to seek appropriate clinical laboratory criteria. The validation process would delineate reference values in vulnerable older adults relative to young and middle aged groups with a view to enable assessment, management and monitoring by diagnostic methods.
\end{abstract}

Keywords: Aging, already known methods, Clinical practice, CVD complication, Diabetes mellitus, Oxidative stress markers, Pathology evidence-base

\section{INTRODUCTION}

\section{Effect of aging on the body's biochemistry and physiology}

Aging is associated with gradual decrease in physical activities, secretion of most hormones and rate of energy generation. ${ }^{1}$ Aging also down regulates lipolysis, ${ }^{2,3}$ which can lead to obesity and metabolic syndrome. Organs such as the liver and pancreas, also undergo degenerative changes, ${ }^{1}$ that are possibly associated development of chronic diseases such as cardiovascular disease (CVD), renal impairment and Type 2 Diabetes Mellitus (T2DM). ${ }^{2}$ The physio-biochemical changes associated with aging are thus complicated and may not be fully explained by just a simple process. Nevertheless, the involvement of oxidative stress as an effect on aging is not in doubt.

Effort has been made to explain how circadian rhythms affect several processes in the body physiology and how diseases that may seem unrelated, such as bone metabolism and vasculopathy, have an increase in oxidative damage as a common underlying biochemistry. ${ }^{4}$ 
Obesity and reduced bone metabolism are interrelated and associated with aging, ${ }^{5}$ as well as circadian rhythm. Further, it is advocated that resetting of the circadian rhythm is a potential way to increase life span and wellbeing. The relation between circadian rhythm and lower energy intake is also explained with regard to the metabolic basis of aging attenuation. ${ }^{6}$ Yet, the mechanism of how hunger can prolong life is not clear. ${ }^{7}$ Perhaps, what is needed is pathology-based evidence for management and monitoring of medical nutritional therapy.

The fact that there is 'increased vulnerability of older adults to DM and its CVD complications' and the additive contribution of oxidative stress from cigarette smoke has also been established. These pieces of knowledge are demonstrated by their inclusions in all cardiovascular complication screening models. ${ }^{8-10}$ However, a gap in Knowledge, Attitude and Practice (KAP) is the lack of considering oxidative stress in persons who are non-smokers but may be suffering stress. ${ }^{11}$ Therefore, the focus of this hypothesis is how to develop pathology evidence-base for oxidative stress in patients regardless of smoking status.

\section{OXIDATIVE STRESS FACTOR BIOCHEMICAL BASIS OF METABOLISM}

IN

\section{Biochemical basis of metabolism in aging}

The cellular basis of aging includes a concept called the 'membrane hypothesis of aging', which involves biochemical processes. ${ }^{12}$ A major feature of the concept is that cells are constantly under-going damage as a result of endogenous oxidative stress agents and heat from exothermic processes. ${ }^{13}$ The physiological process to satisfy cellular energy requirements feed-forward to a sequence of hormonal secretion that stimulates blood glucose metabolism, which in turn affects antioxidant activities and pathophysiology.

In an attempt to explain aging and the patho-biochemistry of various diseases, there is the Advanced Glycation End products (AGE) hypothesis. ${ }^{14}$ There is also the mitochondrial free radical theory that is based on oxidative damage to mitochondrial DNA (mtDNA); ${ }^{15}$ as well as the redox stress hypothesis based on endogenous oxidants. ${ }^{16}$ It is accepted that overproduction of reactive oxygen species in the mitochondria causes mutation of the organelle and that mtDNA mutations accumulate with age. ${ }^{1}$ These hypotheses are inter-linked and implicated in cardiovascular complication of diabetes. Further, various biomarkers have been identified. While the known biomarkers that can be adopted for pathology evidencebased clinical practice are yet to be validated, more novel indicators are still being discovered and demanding a paradigm shift. ${ }^{17}$

What is yet to be seriously discussed is validation of known biomarkers in the context of bringing oxidative stress research to clinical practice. One of the obvious problems is that virtually all reagents used in oxidative stress research are not for in vitro diagnostic use, which casts a question on good laboratory practice when trying to validate such reagents without the manufacturer's approval. A second obvious problem is that some of the biomarkers of aging are either already available, or can be developed in-house, in clinical practice and could constitute oxidative damage indices ${ }^{18}$ but validation as well as determination of reference ranges for older adults relative to young and mid adults is required.

\section{Biochemical basis of metabolism in DM \& CVD}

The hyperglycaemia-induced oxidative stress theory has been put forward to attempt to explain biochemical basis of DM and CVD. Brownlee and Zinman explained how glycolysis is associated with OS via induction of the glycation, hexosamine, polyol and protein kinase $\mathrm{C}$ pathways. ${ }^{19,20}$ There is suggestion that glycolysis is regulated in order to protect against $\mathrm{OS} ;{ }^{21}$ and there is pro-oxidant generation in glycolysis and the Pentose Phosphate Pathway (PPP) when cells are utilizing glucose for the generation of ATP. ${ }^{22}$

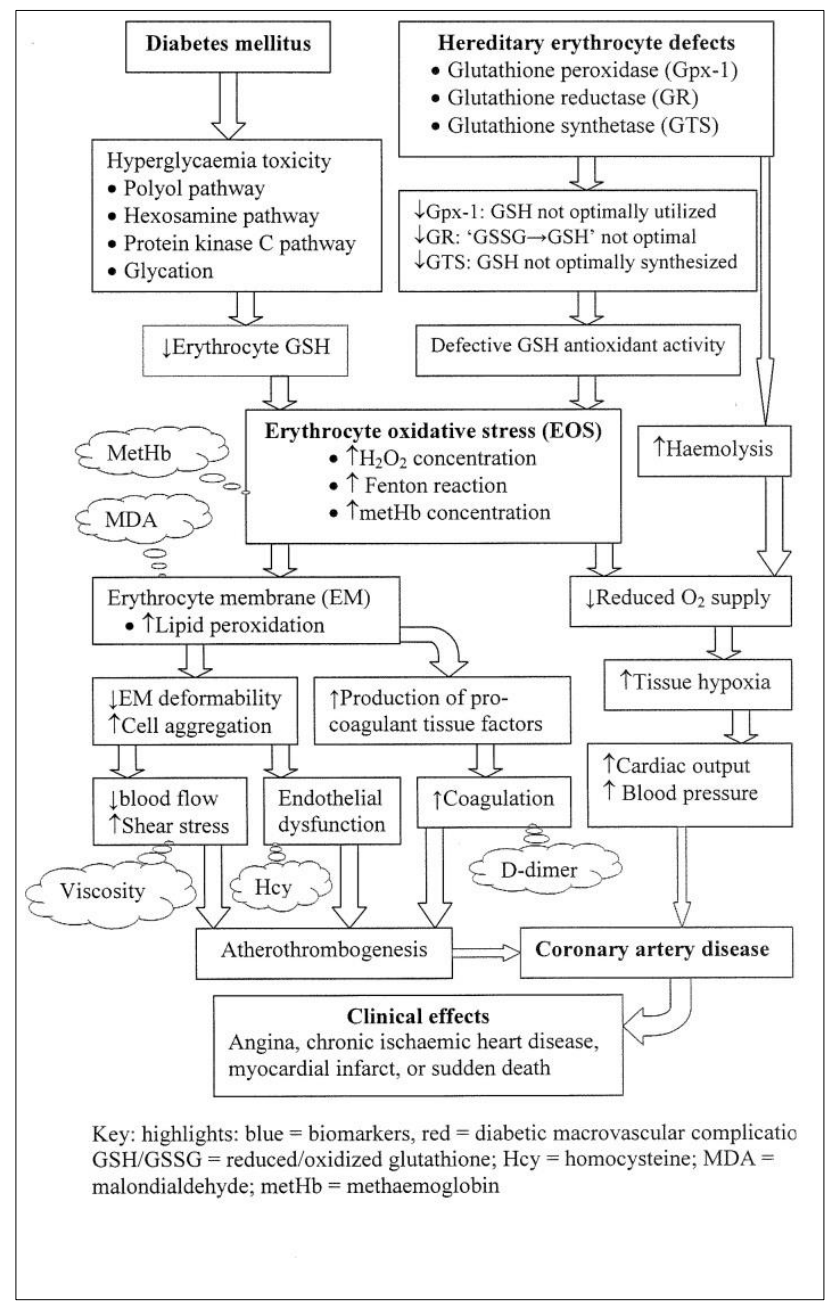

Figure 1: Illustration of links between EOS and related vascular events in diabetes. ${ }^{22}$ 
The antioxidant (GSH) redox cycles depend on the PPP, which produces the reduced form of nicotine adenine diphosphonucleotide (NADPH) and the PPP in erythrocytes is the only source of NADPH, which in turn is the only source of electrons for the regeneration and maintenance of GSH concentration. ${ }^{23}$ Paradoxically though, overproduction of NADPH can drive the increased production of superoxide radical thereby exacerbating OS. ${ }^{24}$ Thus, whether in glycolysis or in PPP, the physiology to meet the cellular need of ATP in the cells such as erythrocyte is associated with the propensity to deplete GSH content, ${ }^{25}$ which in turn leads to oxidative stress. In the red blood cells, the aberrant state of erythrocyte oxidative stress is of clinical importance as illustrated in Figure 1.

\section{Oxidative stress and vitamins $C \& E$ theory of diabetes pathogenesis}

Oxidative stress is a target in the management of diabetic macrovascular complications. ${ }^{22}$ This is associated with the theory that development or progression of diabetes can be respectively delayed or slowed down, and the key to successful prevention and/or treatment is early diagnosis. ${ }^{26}$ Figure 1 illustrates that reduced glutathione (GSH) is the first antioxidant. Beside GSH being a substrate in enzyme catalysed antioxidation, there are other pathways by which it acts as a chain-breaking antioxidant. One pathway is the reduction of metHb to haemoglobin (Figure 2A). This antioxidation reaction is important for the neutralization of the Fenton reaction that will otherwise cause acquired methaemoglobinaemia. ${ }^{27}$ Another major function is the regeneration of other antioxidants including vitamins $\mathrm{C}$ and $\mathrm{E}^{22}$

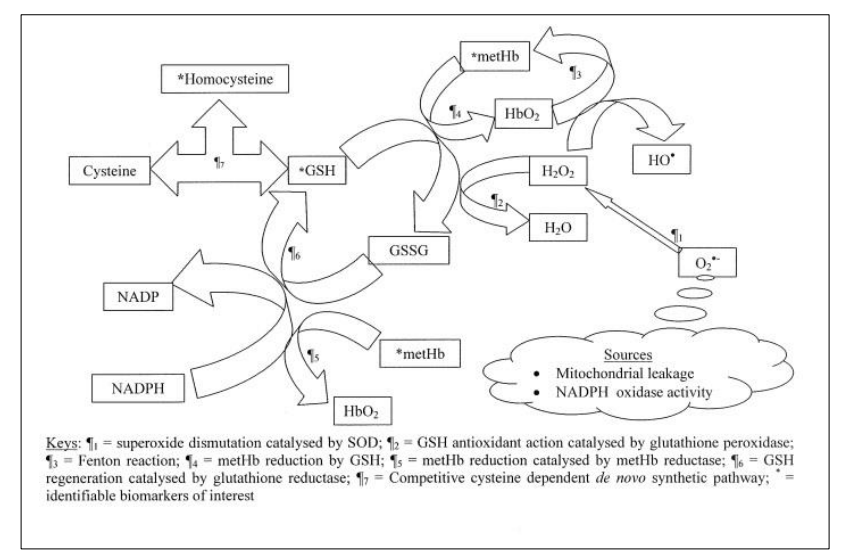

Figure 2A: Antioxidant interactions - illustration of biomarkers and interactions. ${ }^{22}$

When the erythrocyte membrane lipid (L) is oxidized in the process of peroxidation, vitamin E (tocopherol: $\mathrm{TOH}$ ) performs antioxidation by donating one electron (via an $\mathrm{H}^{\bullet}$ radical) to the resultant lipid peroxyl radical (LOO ${ }^{\bullet}$ ) to form lipid peroxide and tocopheroxyl $\left(\mathrm{TO}^{\circ}\right)$ radicals. ${ }^{28}$ The lipid peroxide is further acted upon by GSH, catalysed by Gpx-1, to give a stable lipid-OH. ${ }^{29}$ The antioxidant function of vitamin $\mathrm{C}$ is regeneration of vitamin $\mathrm{E}$ (Equations 1-3), which is also a complex process denoted as 'Vitamin E regeneration system' (VERS; Figure 2B). ${ }^{30,31}$

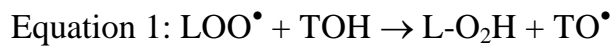

Equation 2: $\mathrm{L}-\mathrm{O}_{2} \mathrm{H}+2 \mathrm{GSH} \rightarrow \mathrm{L}-\mathrm{OH}+\mathrm{GSSG}+\mathrm{H}_{2} \mathrm{O}$

Equation 3: $2 \mathrm{TO}^{\bullet}+$ Ascorbic acid $\rightarrow$ Dehydro-ascorbic acid $+2 \mathrm{TOH}$

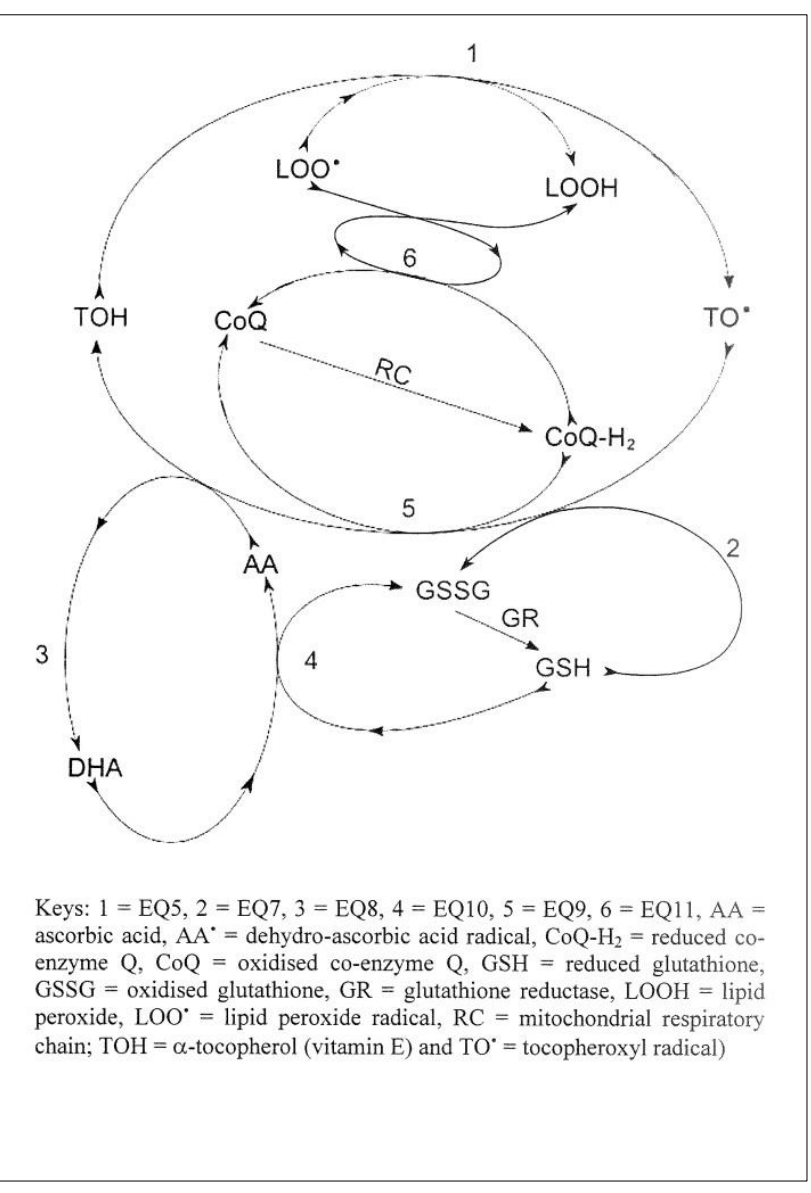

Figure 2B: Antioxidant interactions - vitamin E regeneration system (VERS). ${ }^{30}$

It must be acknowledged that in appreciation of these simplified equations and interactions, there is the theory that the toxic effect of hyperglycaemia is stopped if vitamins $\mathrm{C}$ and $\mathrm{E}$ are recommended at the onset. ${ }^{32}$ Yet, there is also the controversy over efficacy and toxicity to the extent that Guidelines of the American heart association listed antioxidant vitamin supplements among therapies not recommended for CVD prevention. ${ }^{33}$

The complexity and delicate balance of VERS is that antioxidants and oxidants are involved in competitive, simultaneous interactions. These are influenced by the nature of oxidative injury or a prevailing free radical. 
Hence, it is recommended that assumption of an effective antioxidant therapy may be erroneous. In diabetes, the major oxidative injury arises from depletion of cellular $\mathrm{GSH},{ }^{25}$ which feed-forwards to defective regeneration of vitamins $\mathrm{C}$ and $\mathrm{E}$. Therefore, the 'already identified' issue here is the need for evidence-base to ascertain oxidative injury/stress.

\section{Oxidative stress in 'older' vs. 'younger' adults}

The 'free radical theory of aging' is not disputed but experimental and observational studies have yet to produce consistent results. ${ }^{1}$ Mitochondrial dysfunction is a factor of oxidative stress physiology and is associated with disturbance in glucose metabolism. ${ }^{34}$ The manifestation of such disturbances in 'obese' young adults is indicated by signs of premature aging relative to older adults. ${ }^{34}$ However, antioxidants such as ascorbic acid may alleviate some pathophysiological effects of oxidative stress in older, but not in younger adults; ${ }^{35}$ whereas some exercise therapy has been found to be more effective on the younger adults. ${ }^{36}$

With exercise therapy, there is still the call for research to investigate the types of antioxidant supplementation that physically active older adults may need and this is based on the possible paradox of exercise causing more oxidative stress. ${ }^{37}$ That is, there is the complex pathophysiology that demands pathology evidence-base in the use of exercise therapy for the management of agerelated oxidative stress. Thus, it is hereby advanced and hypothesized that there are oxidative damage indices that could be used for such evidence-base to improve management and monitoring of therapy. These biomarkers are available but not currently used to determine changes associated with macrovascular complications of DM and they include BGL, erythrocyte GSH, plasma D-dimer, plasma homocysteine, erythrocyte malondialdehyde and whole blood viscosity. ${ }^{38}$

\section{THE NEED FOR VALIDATION OF KNOWN OXIDATIVE STRESS BIOMARKERS}

\section{Oxidative damage indices - previous report}

It has been reported that subclinical cardiovascular disease (SCVD), which occurs before the manifestation of obvert cardiovascular complications of diabetes, is associated with oxidative damage. In a study that investigated biomarkers associated with diabetes macrovascular complications and erythrocyte oxidative stress implications to determine a set of indices that could be useful to assess oxidative damage in the disease pathogenesis: 266 participants were categorized into control, DM, family history of diabetes, prediabetes, and 'DM+CVD' among others based on participants' clinical history/status. Levels of blood glucose and cholesterol profile, as well as D-dimer, erythrocyte glutathione (GSH), homocysteine, malondialdehyde, and methaemoglobin were determined. Prevalence of significant biomarkers was assessed following a 3.5-year retrospective study. Multivariate analysis showed significant differences between groups $(\mathrm{P}<0.0001)$ with post hoc tests identifying blood glucose level, D-dimer, GSH, and total cholesterol as important variables. Of the subjects who showed hyperglycaemia-associated progression, $89 \%$ had low-level GSH and $44 \%$ had highlevel D-dimer. Some of the participants exhibited prediabetes status and thus qualified for early CVD intervention. $^{18}$

Based on hyperglycaemia-induced oxidative stress being implicated as a cause of increased whole blood viscosity (WBV), which is a clinically modifiable risk factor for CVD: whether or not there is variation in WBV at different stages of DM was investigated in another analysis involving health control, prediabetes, DM and DM+CVD groups. ${ }^{39}$

The prevalence of oxidative stress, indicated by abnormal levels of erythrocyte glutathione, malondialdehyde and methaemoglobin, associated with high WBV was evaluated. The results showed blood viscosity was significantly lower in the control group relative to the prediabetes group; and $\geq 76 \%$ prevalence of oxidative stress was associated with high WBV. It was therefore suggested to redefine the criteria and use WBV on the basis of sensitivity to underlying oxidative stress, instead of neglecting this parameter because of non-specificity to a particular disease condition. The significance is that this easily performed test is an option to consider in an allinclusive laboratory approach to early intervention against future diabetic macrovascular complications. ${ }^{39}$ In the current perspective, this is particularly important for aging individuals with subclinical hyperglycaemia and vasculopathy. More importantly, the results of the studies indicated that GSH level that could be developed inhouse and along with readily available diagnostic tests could constitute oxidative damage panel of markers for assessment, management and monitoring of DM and its cardiovascular complications. Hence, this proposal is to validate the already known panel of biomarkers that can assist in pathology evidence-based pharmacological intervention with anti-aggregation and/or antioxidant agents against future CVD in diabetes. ${ }^{18}$

The major contention here is that new biomarkers are being sought, whereas the basic 'already known' parameters are yet to be adopted. It has previously been noted that there seems to be a paradigm shift from oxidative stress to nitrosative stress, whereas the two concepts are inseparable; just as some researchers have postulated antioxidant properties of apparent metabolic waste products (bilirubinaemia and uricaemia) without proposing adoptability. ${ }^{17}$ Therefore, what is to be validated for adoption in clinical practice is assessment of oxidative damage indices including definition and delineation of how these in older adults compare to stratified younger age groups. 
This concern is of particular importance to low-mid income communities where resources are abysmal. For instance, the SCVD indices available in clinical practice are still limited to big and medium sized or reference laboratories in the mid-high income countries. The new biomarkers that are being researched would hardly be affordable or available to the low-mid income community even when adopted in the distant future. Yet, there are already known methods that could enable remote facilities in the low-mid income countries to run such pathology evidence-base assessment tests.

\section{HYPOTHESIS AND PROPOSAL}

\section{Hypothesis}

This hypothesis borders on 'knowledge, attitude and practice' gap in the context of pathology evidence-base assessment and monitoring of diabetes and its cardiovascular complications. It is based on the opinion that so much is already known, but 'how much of what we already know is practiced?' OS in aging is a compounding factor in increasing vulnerability of older adults to diseases including DM and its CVD complications, but pathology evidence-base assessment of OS is yet to be part of clinical practice in diabetes management. Exercise therapy is known to have paradoxical effects of either alleviating or exacerbating OS and the negative effect can be alleviated by choice of anti-oxidant supplementation; but there need to be an evidence-based assessment, management and monitoring tool. Biomarkers such as erythrocyte GSH, methaemoglobin, plasma D-dimer, plasma homocysteine, erythrocyte malondialdehyde (MDA) and WBV are affordable and can be utilised in the above-mentioned management. However these need to be validated and reference ranges for older adults delineated. It is hereby summarily hypothesized 'on the basis of aging-induced oxidative stress as well as the vitamins C \& E theory of diabetes pathogenesis that:

i. The levels of already known biomarkers of oxidative stress panel (OSP) indices are higher in apparently healthy older adults compared to mid and young adult.

ii. Due to individual differences, retrospective review of pathology data of individuals would reveal lower levels of OSP indices at younger age, if the individual identifies two or more results taken at different apparently healthy points in lifespan.

iii. Upon definition of critical or normal reference values, clinical pathology results of OSP indices taken before and after any intervention would differ.

In advancing these hypotheses, it is noteworthy that questions have been cast over the impression of disagreement between experimental and observational studies' reports vis-a-viz whether there is any observational study with negative result that has actually replicated the methods of an experimental study with positive result. ${ }^{40}$ It is also worthy to note that 'apparently healthy' in this context requires consideration of herbal and over-the-counter non-prescription drugs that the individual may be taking.

\section{Proposal of study setting and outcome}

Participants: Adulthood in the clinical context can be categorized into three age strata including young (20-39), mid (40-65) and older (>65) years old adults. ${ }^{1}$ Setting: The study is proposed to be clinical laboratory based - a translational research per se. Method: The first hypothesis shall be a cross-sectional study and the second could be either a retrospective review of archived clinical pathology data or a small sample sized prospective study. The third would be a prospective clinical observation study. Outcome: The overreaching outcome is development and validation of laboratory test parameters that are useful as evidence for assessment, management and monitoring of risk of DM and it CVD complications among older adults.

\section{CONCLUSION}

This commentary has presented oxidative stress as biochemical and physiological process that underpins aging. The need for validation of 'already known' biomarkers for pathology evidence-base practice is put into the context of ongoing call for improved assessment of increased vulnerability of older adults to diabetes mellitus and its cardiovascular complications.

\section{Funding: No funding sources \\ Conflict of interest: None declared \\ Ethical approval: Not required}

\section{REFERENCES}

1. VanPutte C, Regan J, Russo A. Aging. In: VanPutte C, Regan J, Russo A, eds. Seeley's Anatomy \& Physiology. 9th ed. United States of America: McGraw Hill; 2011.

2. Coppack SW, Jensen MD, Miles JM. In vivo regulation of lipolysis in humans. J Lipid Res. 1994;35(2):177-93.

3. Large V, Arner P. Regulation of lipolysis in humans. Pathophysiological modulation in obesity, diabetes, and hyperlipidaemia. Diabetes Metab. 1998;24(5):409-18.

4. Richards RS, Nwose EU, Bwititi P. Biochemical basis of circadian rhythms and diseases: with emphasis on post-traumatic stress disorder. Med Hypotheses. 2011;77(4):605-9.

5. Cao JJ. Effects of obesity on bone metabolism. J Orthop Surg Res. 2011;6:30. 
6. Froy O, Miskin R. Effect of feeding regimens on circadian rhythms: implications for aging and longevity. Aging. 2010;2(1):7-27.

7. Bubenik GA, Konturek SJ. Melatonin and aging: prospects for human treatment. J Physiol Pharmacol. 2011;62(1):13-9.

8. New Zealand Guidelines Group. New Zealand cardiovascular risk charts, 2009. Available at: http://www.health.govt.nz/publication/new-zealandcardiovascular-risk-charts. Accessed 28 April 2014.

9. The Framingham Study. Cardiovascular disease (10year risk), 2014. Available at: http://www.framinghamheartstudy.org/riskfunctions/cardiovascular-disease/10-year-risk.php. Accessed 28 March 2014.

10. BUPA. How does your health age stack up against your real age? BUPA Australia Pty Ltd, 2010. Available at: http://www.bupa.com.au/health-andwellness/tools-and-apps/tools-and-calculators/quickhealth-age-check. Accessed 26 December 2013.

11. Bwititi PT, Nwose EU. Screening of cardiovascular disease risk in diabetes: questions concerning prediabetes and low-mid income countries. North Am J Med Sci. 2014;6(6):284-6.

12. Zs-Nagy I. Aging of cell membranes: facts and theories. Interdiscipl Topics Gerontol. 2014;39:6285.

13. Zs-Nagy I. On the role of intracellular physicochemistry in quantitative gene expression during aging and the effect of centrophenoxine. A review. Arch Gerontol Geriatr. 1989;9(3):215-29.

14. Krautwald M, Munch G. Advanced glycation end products as biomarkers and gerontotoxins - a basis to explore methylglyoxal-lowering agents for Alzheimer's disease? Exp Gerontol. 2010;45(10):744-51.

15. Jenny NS. Inflammation in aging: cause, effect, or both? Discov Med. 2012;13(73):451-60.

16. Sohal RS, Orr WC. The redox stress hypothesis of aging. Free Radic Biol Med. 2012;52(3):539-55.

17. Nwose EU, Bwititi PT, Chalada MJ. Oxidative stress research: a framework to relate basic science to clinical practice. Oxid Antioxid Med Sci. 2013;2(4):225-9.

18. Nwose E, Richards R, Kerr P, Tinley R, Jelinek H. Oxidative damage indices for the assessment of subclinical diabetic macrovascular complications. Br J Biomed Sci. 2008;65(3):136-41.

19. Brownlee M. The pathobiology of diabetic complications: a unifying mechanism. Diabetes. 2005;54(6):1615-25.

20. Zinman B. Pathways leading to diabetic microvascular complications and the latest therapies CME, 2003. Available at: http://www.medscape.com/viewprogram/2636?src= search. Accessed 12 April 2007.

21. Bensaad K, Tsuruta A, Selak MA, Vidal MN, Nakano K, Bartrons R, et al. TIGAR, a p53inducible regulator of glycolysis and apoptosis. Cell. 2006;126(1):107-20.
22. Nwose EU, Jelinek HF, Richards RS, Kerr PG. Erythrocyte oxidative stress in clinical management of diabetes and its cardiovascular complication. $\mathrm{Br} \mathrm{J}$ Biomed Sci. 2007;64(1):35-43.

23. McMullin MF. The molecular basis of disorders of the red cell membrane. J Clin Pathol. 1999;52(4):245-8.

24. Boada J, Cuesta E, Riog T, Gámez A, Carbonell T, Ventura F, et al. Enhanced antioxidant defences and resistance to TNF-alpha in a glycolysis-depleted lung epithelial cell line. Free Radic Biol Med. 2002;33(10):1409-18.

25. Ceconi C, Boraso A, Cargnoni A, Ferrari R. Oxidative stress in cardiovascular disease: myth or fact? Arch Biochem Biophys. 2003;420(2):217-21.

26. Bell DSH. Chronic complications of diabetes. South Med J. 2002;95(1):30-4.

27. de Haan JB, Crack PJ, Flentjar N, Iannello RC, Hertzog PJ, Kola I. An imbalance in antioxidant defense affects cellular function: the pathophysiological consequences of a reduction in antioxidant defence in glutathione peroxidase-1 (Gpx-1) knockout mouse. Redox Rep. 2003;8(2):6979.

28. Young IS, Woodside JV. Antioxidants in health and disease. J Clin Pathol. 2001;54(3):176-86.

29. de Haan JB, Witting PK, Stefanovic N, Pete J, Daskalakis M, Kola I, et al. Deficiency in the antioxidant enzyme glutathione peroxidase-1 (Gpx1) does not increase atherosclerosis in C57BL/J6 mice fed a high fat diet. J Lipid Res. 2006;47(6):1157-67.

30. Nwose EU, Jelinek HF, Richards RS, Kerr PG. The 'vitamin E regeneration system' (VERS) and an algorithm to justify antioxidant supplementation in diabetes - a hypothesis. Med Hypotheses. 2008;70(5):1002-8.

31. Kagan VE, Tyurina YY. Recycling and redox cycling of phenolic antioxidants. Ann NY Acad Sci. 1998;854(1):425-34.

32. Baker L. Antioxidants taken with junk food can make it safer. (UniSci Daily), 2002. Available at: http://unisci.com/stories/20022/0617021.htm. Accessed 4 June 2008.

33. Mosca L, Banka CL, Benjamin EJ, Berra K, Bushnell C, Dolor RJ, et al. Evidence-based guidelines for cardiovascular disease prevention in women: 2007 update. Circulation. 2007;115(11):1481-501.

34. Niemann B, Chen Y, Teschner M, Li L, Silber RE, Rohrbach S. Obesity induces signs of premature cardiac aging in younger patients: the role of mitochondria. J Am Coll Cardiol. 2011;57(5):57785.

35. Monahan KD, Eskurza I, Seals DR. Ascorbic acid increases cardiovagal baroreflex sensitivity in healthy older men. Am J Physiol Heart Circ Physiol. 2004;286(6):H2113-7.

36. Johnson ML, Lanza IR, Short DK, Asmann YW, Nair KS. Chronically endurance-trained individuals 
preserve skeletal muscle mitochondrial gene expression with age but differences within age groups remain. Physiol Rep. 2014;2(12):pii: e12239.

37. Ji LL. Exercise at old age: does it increase or alleviate oxidative stress? Ann N Y Acad Sci. 2001;928:236-47.

38. Ridker PM, Brown NJ, Vaughan DE, Harrison DG, Mehta JL. Established and emerging plasma biomarkers in the prediction of first atherothrombotic events. Circulation. 2004;109(25 Suppl 1):IV6-19.

39. Richards RS, Nwose EU. Blood viscosity at different stages of diabetes pathogenesis. $\mathrm{Br} \mathrm{J}$ Biomed Sci. 2010;67(2):67-70.
40. Nwose EU. Towards optimizing medical nutrition therapy with laboratory methods. In: Nwose EU, eds. Oxidative Stress Concepts in Clinical Practice. 10th ed. Germany: LAP Lambert Academic Publishing; 2010.

DOI: 10.5455/2320-6012.ijrms20150359

Cite this article as: Nwose EU, Bwititi PT, Richards RS. Increased vulnerability of older adults to DM and its CVD complications: proposal for assessment, management and monitoring by diagnostic methods. Int J Res Med Sci 2015;3:810-6. 